\title{
The "bald" right atrium: an unexpected approach
}

\author{
Márcio Galindo Kiuchi* ${ }^{* 1}$ and Shaojie Chen ${ }^{2}$ \\ ${ }^{1}$ Division of Cardiac Surgery and Artificial Cardiac Stimulation, Department of Medicine, Hospital e Clínica São Gonçalo, Brazil \\ ${ }^{2}$ Department of Cardiology, Shanghai First People's Hospital, Shanghai Jiao Tong University School of Medicine, Shanghai, China
}

\section{Case report}

Pacing in the right atrium may induce atrial dyssynchrony and subsequent AF [1]. The percentage of atrial pacing can lead to hemodynamic alterations, and changes in the shape and velocity of intra-atrial, interatrial, and atrioventricular conduction, as well as alter cellular properties. An association between \%AP and AF may be possible because of differences in baseline variables. Because all of the patients in this study had this feature turned on, we are not able to confirm this possibility. Pacing strategies targeting the intra- and interatrial conduction tracts $[2,3]$ and algorithms aimed at minimizing atrial pacing should be further examined.

In our case report, we presented a female patient, 64 years old, complaining of syncope, pre-syncope, and fatigue on usual efforts. It has been ablated to atrium-ventricular node reentrant tachycardia

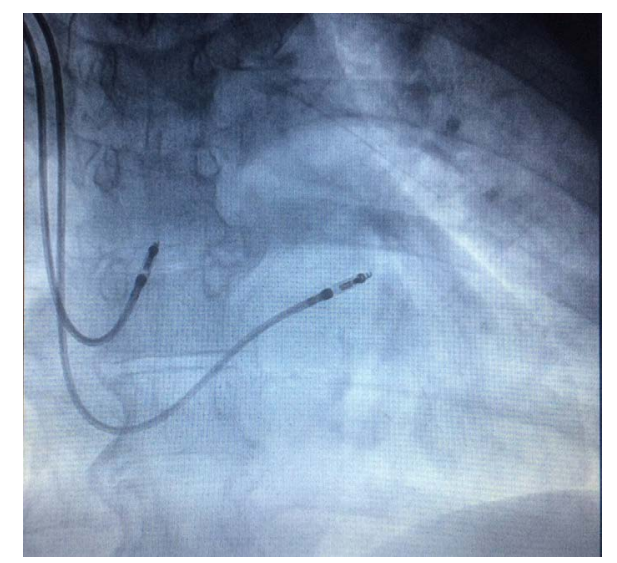

Figure 1. The right atrial lead positioning observed by a posterior-anterior view no acceptable sense and threshold were registered in the several positions (the "bald" right atrium).

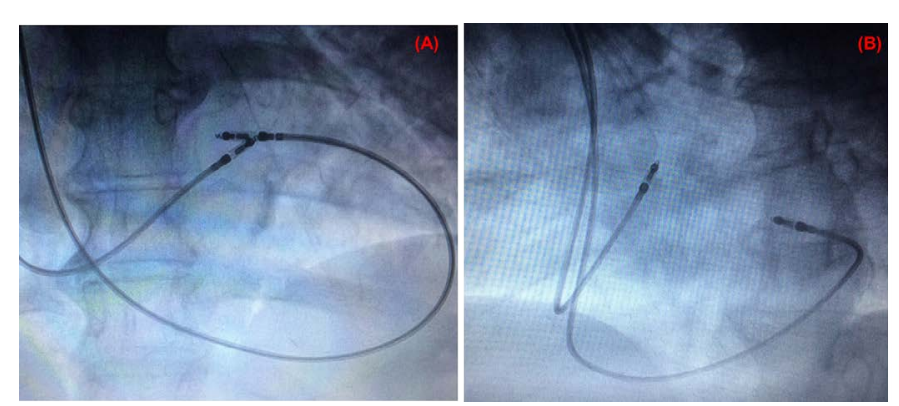

Figure 2. Atrial lead into the coronary sinus, positioning it in the in the highest position possible (A) postero-anterior view and (B) left-anterior oblique view, fixing it in this position and measuring the left atrial parameters through the coronary sinus.
(AVNRT) and is in use of sotalol hydrochloride160 mg twice daily for the treatment of ventricular premature beats. She has hypertension, type 2 diabetes mellitus, dyslipidemia, treated with losartan $100 \mathrm{mg}$ daily, metformin hydrochloride 850 twice a day, and rosuvastatin 10 mg daily. She also has negative myocardial scintigraphy for ischemia, normal carotid Doppler and echocardiogram, normal 24-hour Holter monitoring showing heart rate (minimum, average and maximum) of 58-78-122 bpm, sinus rhythm, 6633 polymorphic ventricular premature beats, 2 episodes of non-sustained ventricular tachycardia composed by 3 beats fast as $128 \mathrm{bpm}$. Cardiac nuclear magnetic resonance image showing left ventricular ejection fraction $60 \%$, an end-diastolic diameter of $48 \mathrm{~mm}$, and without necrosis or ischemia. She underwent electrophysiologic study that showed dysfunction of the distal electrical conduction, with HV interval of $78 \mathrm{~ms}$. Faced with this situation, the dual chamber pacemaker was indicated.

During the positioning of the right ventricular lead in the middle interventricular septum, no change was noted, being fixed and having the following parameters: $\mathrm{R}$ wave $=12.0 \mathrm{mV}$, impedance $=620 \Omega$, and threshold: 0.5 V@0.5 ms. However, during the right atrial lead positioning observed by a posterior-anterior view (Figure 1) no acceptable sense and threshold were registered in the several positions (the "bald" right atrium): presenting $\mathrm{P}$ wave measures $<0.5 \mathrm{mV}$ and thresholds>3.5 V@1.0 ms. Given this problem, we opted to insert the atrial lead into the coronary sinus, positioning it in the in the highest position possible, fixing it in this position and measuring the left atrial parameters through the coronary sinus observed by a posterior-anterior view and left-anterior oblique view (Figure $2 \mathrm{~A}$ and $\mathrm{B}$ ), which were the following: $\mathrm{P}$ wave $=3.2 \mathrm{mV}$, impedance $=850 \Omega$, and threshold: 0.9 V@0.5 ms. The leads were connected to DDDR generator, and the incision was sutured and closed in layers. The pacemaker was finally programmed with the following atrial and ventricular parameters, respectively: bipolar sense presenting $\mathrm{P}$ wave $=3.4 \mathrm{mV}$, impedance $=670 \Omega$, left atrial threshold =0.9 V@0.5 ms, and unipolar output = $2.5 \mathrm{~V} @ 0.5 \mathrm{~ms}$; bipolar sense presenting R wave $=14.2 \mathrm{mV}$, impedance $=590 \Omega$, ventricular threshold $=0.4 \mathrm{~V} @ 0.5 \mathrm{~ms}$, and and bipolar output $=2.0 \mathrm{~V} @ 0.5 \mathrm{~ms}$.

In this case, we can conclude that despite the difficulties encountered to position the lead in the right atrium, we found an alternative way to sense and pacing the left atrium, by the intra coronary sinus approach.

Correspondence to: Márcio Galindo Kiuchi, MD, MPH, Rua Cel. Moreira César 138 - Centro, São Gonçalo - Rio de Janeiro - Brazil; E-mail: skales@hsph. harvard.edu

Received: October 01, 2016; Accepted: October 13, 2016; Published: October 17,2016 


\section{References}

1. Andersen HR, Nielsen JC, Thomsen PE, Thuesen L, Mortensen PT, et al. (1997) Longterm follow-up of patients from a randomised trial of atrial versus ventricular pacing for sick-sinus syndrome. Lancet 350: 1210-1216. [Crossref]

2. Dabrowska-Kugacka A, Lewicka-Nowak E, Rucinski P, Zagozdzon P, Raczak G, et al. (2009). Atrial electromechanical sequence and contraction synchrony during single- and multisite atrial pacing in patients with brady-tachycardia syndrome. Pacing Clin Electrophysiol 32: 591-603.

3. Dabrowska-Kugacka A, Lewicka-Nowak E, Rucinski P, Kozlowski D, et al. (2010). Single-site Bachmann's bundle pacing is beneficial while coronary sinus pacing results in echocardiographic right heart pacemaker syndrome in brady-tachycardia patients. Circ J 74: 1308-1315.

Copyright: (C2016 Kiuchi MG. This is an open-access article distributed under the terms of the Creative Commons Attribution License, which permits unrestricted use, distribution, and reproduction in any medium, provided the original author and source are credited. 